\title{
Dielectric elastomer generator with equi-biaxial mechanical loading for energy harvesting
}

\section{Citation}

Jiangshui Huang, Samuel Shian, Zhigang Suo, David R. Clarke. 2013. "Dielectric Elastomer Generator with Equi-biaxial Mechanical Loading for Energy Harvesting." Presented at Electroactive Polymer Actuators and Devices (EAPAD) 2013, March 10, 2013, San Diego, California. In Proceedings of SPIE 8687, 86870Q.

\section{Published Version}

doi:10.1117/12.2009724

\section{Permanent link}

http://nrs.harvard.edu/urn-3:HUL.InstRepos:12965659

\section{Terms of Use}

This article was downloaded from Harvard University's DASH repository, and is made available under the terms and conditions applicable to Open Access Policy Articles, as set forth at http:// nrs.harvard.edu/urn-3:HUL.InstRepos:dash.current.terms-of-use\#OAP

\section{Share Your Story}

The Harvard community has made this article openly available.

Please share how this access benefits you. Submit a story.

\section{Accessibility}




\title{
Dielectric Elastomer Generator with Equi-biaxial Mechanical Loading for Energy Harvesting
}

\author{
Jiangshui Huang*, Samuel Shian*, Zhigang Suo ${ }^{a}$, David R. Clarke ${ }^{\text {b }}$ \\ School of Engineering and Applied Sciences, Harvard University, \\ Cambridge, MA 02138
}

\begin{abstract}
Dielectric elastomer generators (DEGs) are attractive candidates for harvesting electrical energy from mechanical work since they comprise relatively few moving parts and large elastomer sheets can be mass produced. Successfully demonstrations of the DEG prototypes have been reported from a diverse of energy sources, including ocean waves, wind, flowing water and human movement. The energy densities achieved, however, are still small compared with theoretical predictions. We show that significant improvements in energy density (550 J/kg with an efficiency of $22.1 \%$ ), can be achieved using an equi-biaxial mechanical loading configuration, one that produces uniform deformation and maximizes the capacitance changes. Analysis of the energy dissipations indicates that mechanical losses, which are caused by the viscous losses both within the acrylic elastomer and within the thread materials used for the load transfer assembly, limits the energy conversion efficiency of the DEG. Addressing these losses is suggested to increase the energy conversion efficiency of the DEG. .
\end{abstract}

Keywords: Dielectric, Elastomers, Generator, Electromechanical, Capacitance, Viscosity, Energy

[*] These authors contributed equally to this work. [a]. suo@seas.harvard.edu, phone 617-495-3789; [b]. clarke@ seas.harvard.edu, phone 617-495-4140 


\section{INTRODUCTION}

A dielectric elastomer generator (DEG) is, in essence, a variable capacitor that converts stored mechanical or elastic energy into electrical energy by transferring charges from low to high potential. The mechanical action that changes DEG capacitance can be achieved using several methods, including direct linear motion, simplifying the number of moving parts. DEGs are attractive candidates for harvesting electrical energy at large scale since large elastomer sheets can be mass produced at relatively low cost. Demonstrations of harvesting electrical energy from diverse energy sources, including ocean waves, wind, flowing water and human movement have been reported. ${ }^{1-7}$ Also demonstrated are charging small electrical devices such as cell phones and sensors embedded in human body for medical applications. ${ }^{1,2}$, 4-7 Although various prototypes using different materials and various harvesting circuits and cycles have been reported in the literature since DEGs were first proposed in $2001,{ }^{1}$ the energy densities achieved to date are still far less than the theoretical prediction of $1.7 \mathrm{~J} / \mathrm{g}$ based on acrylic elastomers. ${ }^{1-16}$ For example, the ocean wave energy harvesting buoy has an energy density of about $135 \mathrm{~J} / \mathrm{kg}$, the DEG with balloon geometry has an energy density of $102 \mathrm{~J} / \mathrm{kg}$, and the self-priming DEGs have an energy density from $2.8 \mathrm{~J} / \mathrm{kg}$ to $12.6 \mathrm{~J} / \mathrm{kg} .{ }^{3,7,11-13,15,16}$ A relatively high energy density, 0.3 $\mathrm{J} / \mathrm{g}$, has been reported for shoe generators, ${ }^{15}$ but the significance of this value is difficult to evaluate due to limited technical details reported. As we will show, considerably higher energy densities can be achieved using equi-biaxial mechanical loading since this configuration maximizes the capacitance changes and hence the electrical energy generated in a mechanical cycle.

(a)

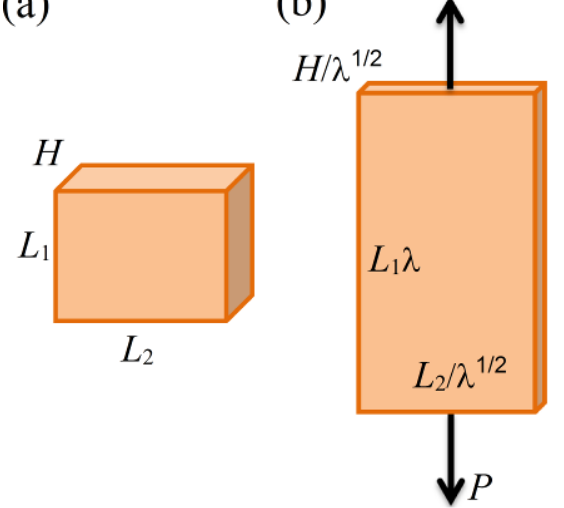

(b)

Reference State Uniaxial Stretch (c)

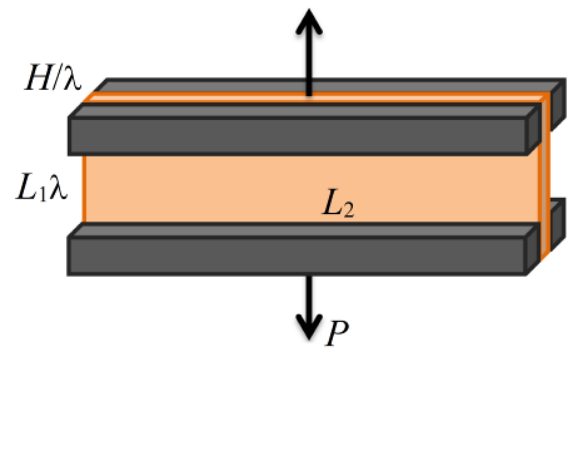

Pure Shear Stretch (d)

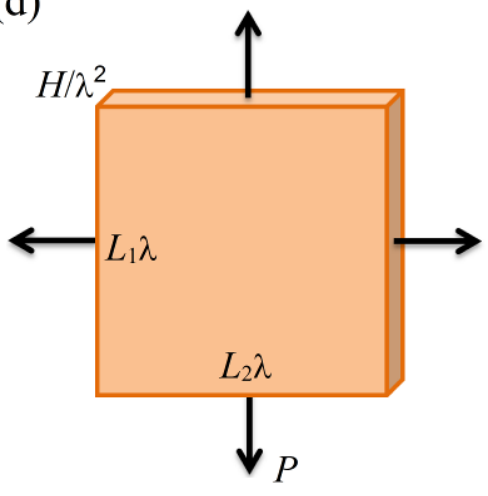

Equi-biaxial Stretch

Figure 1. (a) An elastomer of length $L_{1}$, width $L_{2}$, and thickness $H$ at reference state was stretched under different mechanical loading configurations: (b) By uniaxial stretch; the stretch of length $\left(L_{l}\right)$ is $\lambda$, while both the stretches of width and thickness are $\lambda^{-1 / 2}$; (c) By pure shear stretch if $L_{1} \ll L_{2}$; the stretch of length $\left(L_{l}\right)$ is $\lambda$, while the width remains constant, and the stretch of thickness is $\lambda^{-1}$; (d) By equi-biaxial stretch; the stretches of length and width are $\lambda$, while the stretch of thickness is $\lambda^{-2}$. Since capacitance is proportional to the length, to the width, and to the inverse of thickness, the stretch $\lambda$ results in the increase of capacitance which is proportional to $\lambda, \lambda^{2}$, and $\lambda^{4}$ in the uniaxial, pure shear, and equi-biaxial loading configurations, respectively.

Since the electrical energy stored in a capacitor is proportional to the square of the voltage and to the capacitance, the achievable energy density of a DEG depends on the maximum electrical field which the DEG can endure and on how much the capacitance can be changed during an electromechanical cycle. While the former factor is an intrinsic property 
of a dielectric elastomer (DE) material, the latter factor depends strongly on the loading geometry, where the capacitance of the DE increases as it is stretched in area and squeezed in thickness. In Fig. 1, we show that uniaxial, pure-shear, and equi-biaxial stretching configurations increase the capacitance in proportion to the first, second and fourth power of the

stretch, $\lambda$, respectively, where $\lambda$ is defined as the linear length ratio of the deformed and the un-deformed states in the direction of the stretching force. ${ }^{17}$

Another advantage of equi-biaxial loading is in the moving boundary condition imposed over the edges of the materials, which results in relatively uniform deformations over the active elastomer area. Such uniform deformation prevents concentration of electrical field or mechanical stress that may lead to premature failures. ${ }^{7}$ Other loading configuration, such as in case of an elastomer sheet mounted on a chamber of air, has a fixed boundary condition, resulting in an inhomogeneous state of stretch, being equi-biaxial at the center of the sheet and pure-shear at the edge of the sheet. ${ }^{13}$ Here we take advantage of both the high capacitance changes and the uniform elastomer deformations afforded using a setup of equi-biaxial loading. A maximum energy density of $550 \mathrm{~J} / \mathrm{kg}$ has been achieved at $22.1 \%$ efficiency. Although this is far higher than a recent report of an efficiency of $7.5 \%{ }^{13}$ also using an acrylic elastomer, we will show that the efficiency is mainly limited by the viscous losses in the acrylic elastomer, indicating that significantly higher efficiencies could be attainable with other elastomers.

\section{ENERGY HARVESTING CYCLE}

To illustrate the principle of the DEG presented in this paper, and for direct comparison with our observations, a simplified energy harvesting cycle is schematically shown in Fig. 2. This neglects dissipative processes, ${ }^{18}$ the limited harvesting capacitance, and the electric energy consumed by the measurement sub-assemblies. This idealized cycle was theoretically suggested, ${ }^{19}$ and was approximately realized in an experiment using a membrane mounted on a chamber of air. ${ }^{13}$ Our implementation involves transferring electric charge from a power supply of voltage $\Phi_{P S}$ to a large capacitor of a higher voltage of $\Phi_{C}$. Starting in state $A$ with a minimally stretched elastomer at the high voltage of the capacitor, $\Phi_{C}$, and a small charge, $Q_{\text {low }}$, the elastomer is stretched under the open-circuit conditions to state $B$. In doing so, the charge

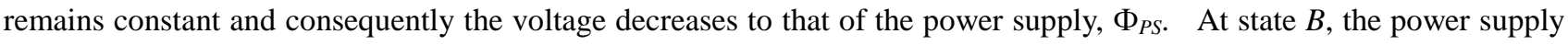
is connected and the stretching is continued to a pre-determined maximum stretch at state $C$. During this step, charge flows from the power supply to the DE electrodes at a constant voltage. When state $C$ is reached, the power supply is disconnected and the stretching forces are decreased from the maximum allowing the elastomer to begin shrinking back elastically. As it does so, its thickness increases, the capacitance consequently decreases and so the voltage increases. At a prescribed voltage, which is equal to the voltage of the capacitor, $\Phi_{C}$, and chosen to avoid dielectric breakdown, the electrodes are connected to the harvesting circuit and as the stretching force is decreased further, the potential across the DE electrode exceeds the voltage on the collecting capacitor causing charge to flow from the elastomer to the collecting circuit. This continues until the elastomer has shrunk back to its original size at state $A$. So, in one complete cycle, a charge, $\Delta Q=Q_{\text {high }}-Q_{\text {low }}$, is boosted from the low voltage $\Phi_{P S}$ to the high voltage $\Phi_{C}$, by converting part of the elastic energy stored in the elastomer. Correspondingly, the net electric energy harvested is $\Delta Q\left(\Phi_{C}-\Phi_{P S}\right)$. The non-linearity of the curves $A B$ and $C D$ in Fig. 2(a) results from the quartic dependence of the capacitance on the stretch. 


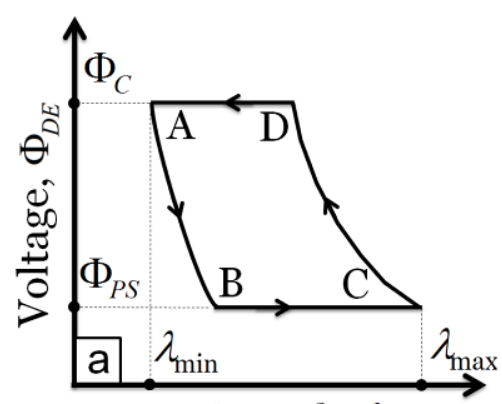

Stretch, $\lambda$

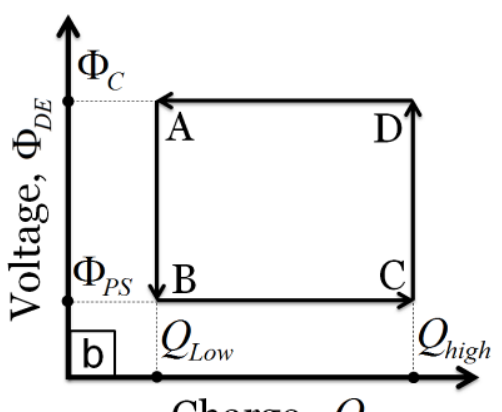

Charge, $Q_{D E}$

Figure 2. Simplified schematics show the principle of the DEG in an energy harvesting cycle: (a) Voltage of DEG vs. Stretch; (b) Voltage of DEG vs. Charge.

\section{EXPERIMENTAL}

In our experiment, a thin sheet of a DE was under cyclic mechanical load and connected to the electrical circuit (Fig. 3). In common with many previous studies of DEGs, thin sheets of acrylic elastomers (VHB 4905, 3M) were used as the dielectric and a layer of carbon grease (CAT. NO. 846-80G, MG Chemicals) was coated on both sides to serve as compliant electrodes. The elastomer thickness was $H=0.5 \mathrm{~mm}$, and the electroded radius, $R_{0}$, was $2.0 \mathrm{~cm}$ corresponding to a generator mass of $M=0.60 \mathrm{~g}$. The mechanical loading configuration was modified from that in our previous work on DE actuators. ${ }^{20}$ Equi-biaxial loading was accomplished by applying radial forces to the circumference of the elastomer sheet through a system of clips, threads and pulleys all loaded by the motion of a linear servo-motor (Model SLP35, Nippon Pulse America Inc.). The applied force, $F$, was recorded with a load cell (Model LSB200, Futek, Inc.) attached to the servo. When the servo was at zero displacement $L=0$, the DE disc was slightly pre-stretched, and the force $F$ was relatively small. As the displacement, $L$, was cyclically increased and decreased, the mechanical energy in each cycle, $\Delta E_{\mathrm{M}}$, was determined by integrating the load-displacement cycle:

$$
\Delta E_{M}=\oint F d L
$$

Simultaneously, the size of the sheet was recorded with a video camera, from which the radius $R$ of the sheet and the radial stretch, defined as $\lambda=R / R_{0}$, were obtained.

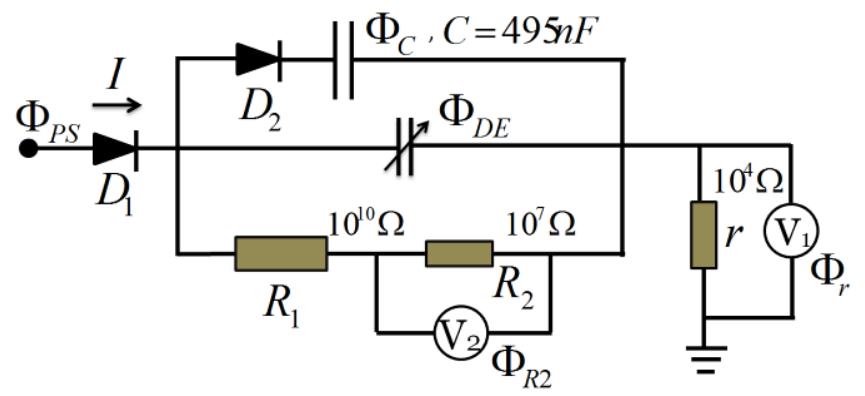

Figure 3. The electrical circuits for energy harvesting and for measuring the voltage and current.

The main components in the circuit used for energy harvesting consisted of a power supply, a large harvesting capacitor, a sheet of DE, and two diodes (Fig. 3). The charge $\left(\Delta Q_{P S}\right)$ at the low voltage $\left(\Phi_{P S}\right)$ was delivered from the power supply (model 610E, Trek Inc.), and the same charge at the high voltage $\left(\Phi_{C}\right)$ was delivered to the capacitor 
assembly (495 $n F$, Part\# C4BSYBX3330ZBLJ, Kemet Corp.) which was relatively larger compared to elastomer capacitance at the maximum stretch $\left(\mathrm{C}_{\mathrm{DE}} \sim 60 \mathrm{nF}\right)$. The diode $D_{1}$ (NTE517, NTE Electronics, Inc) only allowed a charging current to flow when the voltage across the elastomer, $\Phi_{D E}$, was lower than that of the power supply, $\Phi_{P S}$. The diode $D_{2}$ only allowed the charge to flow from the elastomer to the capacitor when $\Phi_{D E}>\Phi_{C}$. The current, $I$, was recorded with a voltmeter, $V_{l}$ (Model 2000, Keithley) in parallel with a resistor, $r$. The voltage across the elastomer, $\Phi_{D E}$, was

measured by a voltmeter, $V_{2}$, (model USB-6218, National Instruments) through a voltage divider, $R_{I}$ and $R_{2}$. Analysis of measurements indicates that these additional components for voltage and current measurements did not appreciably perturb the energy harvesting cycle.

\section{RESULTS AND DISCUSSION}

The energy harvesting cycle of the DEG under equi-biaxial loading for the first three mechanical loading and unloading cycles recorded at $0.5 \mathrm{~Hz}$ and a voltage input, $\Phi_{P S}$, of $2 \mathrm{kV}$, is shown in Fig. 4. They have the same basic harvesting characteristics as the ideal schematic shown in Fig. 2, but there are notable changes as the cycle number increases: The maximum voltage across the elastomer increases, and so do the maximum charge transferred and the maximum stretch of the elastomer. The maximum voltage of the DE in each cycle is corresponding to the voltage of the capacitor $\Phi_{C}$. The increase in the maximum voltage is a direct consequence of using a capacitor of finite capacitance: the unidirectional delivery of charge from the DE to the capacitor in each cycle results in an increase of the voltage of the capacitor. The increases of the maximum charge transferred and the maximum stretch of the elastomer are a direct consequence of the mechanical viscous creep of the elastomer under load and the finite stiffness of the mechanical loading system, which is also evident in the plots of force, voltage, and harvesting current when the servo displacement is prescribed as a function of time for the electro-mechanical cycles (Figure 5a). For instance, as the elastomer elongates under load, the force required to stretch the elastomer to the same maximum stretch decreases. Similarly, as the elastomer creeps viscously with cycling it becomes thinner and its capacitance increases resulting in a larger current flow. Also, due to the viscous creeping, when the servo moved back to the zero displacement, the DE did not immediately recover the minimally stretched state and thus the DE was nearly at a state of loss of tension within a small servo displacement, leading to a delay between the voltage shut-off and the beginning of the next loading cycle. 

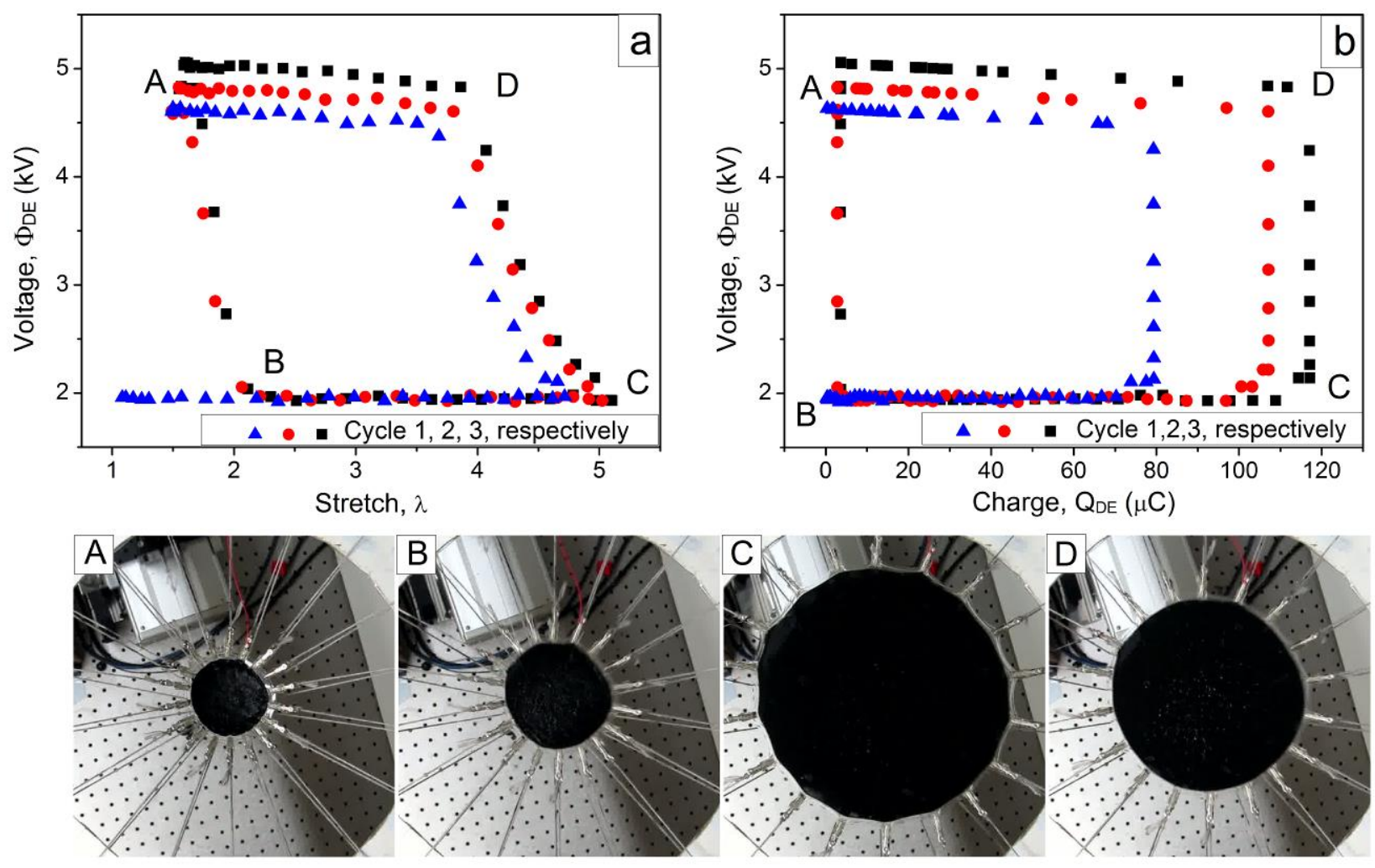

Figure 4. (a) The first three cycles for voltage as a function of stretch (b) The first three cycles for voltage as a function of the charge on DE. "A, B, C, D" in the figures indicate the states of DE in electromechanical cycle corresponding to the schematic diagram in Fig.2. The "first cycle" is not a complete cycle because it did not start at a high voltage (state A), instead it started at the voltage of the power supply (state B). The bottom row figures are four snap-shots corresponding to the states A, B, C and D, as marked in (a) and (b).

After the end of cycles ' 2 ', ' 3 ' and ' 4 ', the maximum voltage of the DE (the voltage of the capacitor) in each cycle increased by over $200 \mathrm{~V}$. During the contraction in the fifth cycle, the voltage initially increased at the same pace as the previous cycles, however, midway through the contraction, the voltage reached a point where the total electric energy equal the elastic strain energy of the DE. In other words, the electrostatic attraction between opposite charges on the electrodes overcome the contractive elastic force of the DE and manifested as a loss of sheet tension or a stagnation of contraction, halting further increase in the DE voltage. After this point, the DE membrane contracted at a slower pace, which is mainly through charge dissipations within the membrane thickness or external circuits. While this recovery stagnation is the basis for DE in actuator mode, however, one should avoid this condition for generator mode because the stored elastic energy effectively cannot be converted into electrical energy.

Despite these non-idealities, the capacitance changes do vary with the fourth power of the biaxial stretch (Fig. 5b). This is consistent with the simple geometric argument that the capacitance varies as the fourth power of the stretch for an incompressible solid under biaxial loading. 
(a)

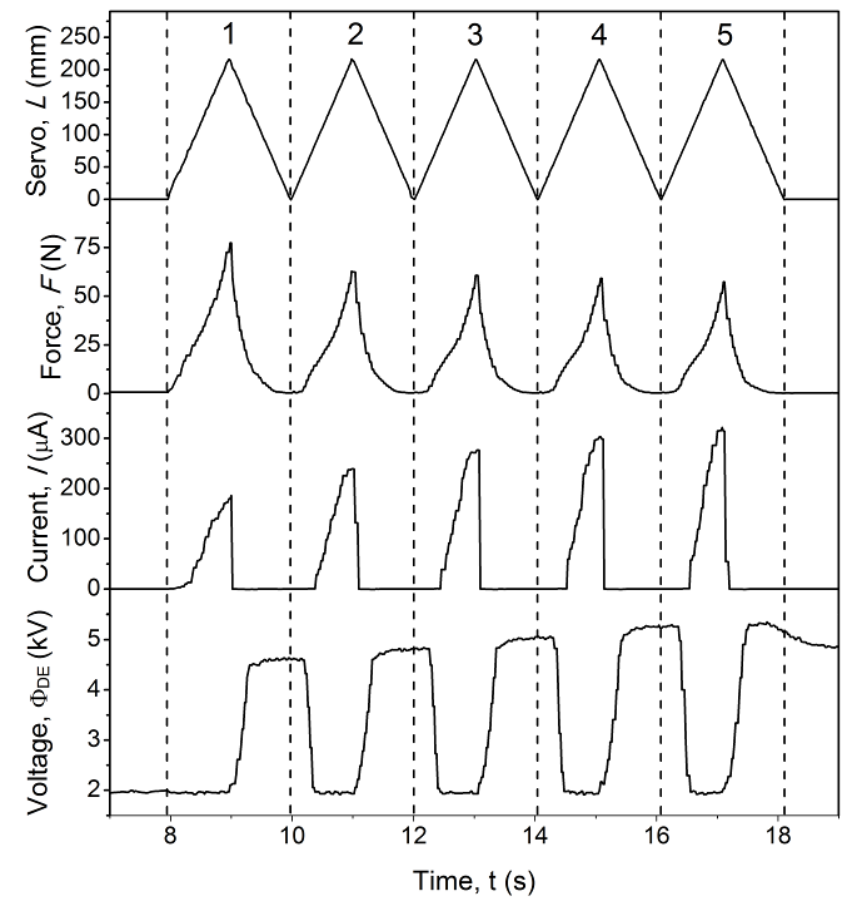

(b)

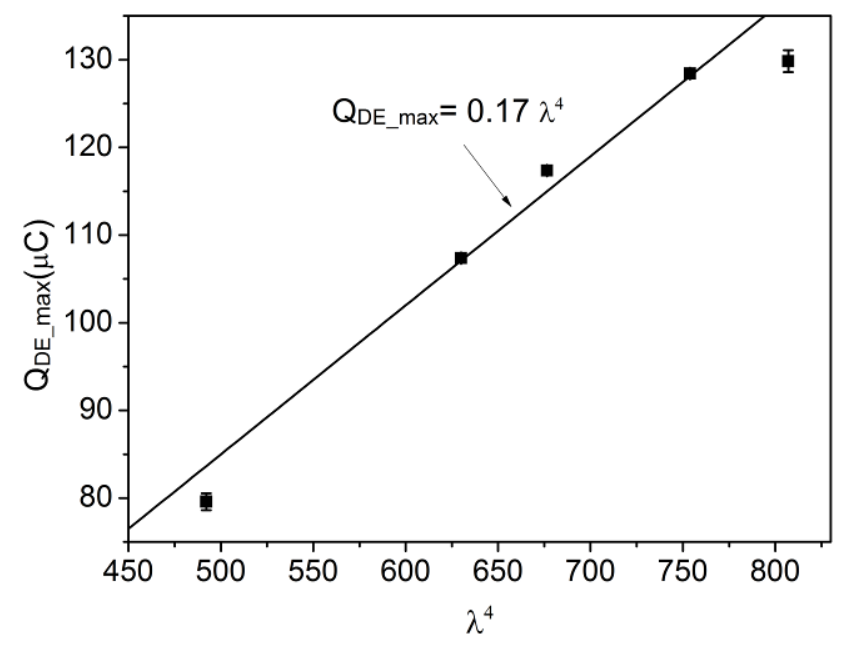

Figure 5. (a) The servo displacement, $L$, the voltage of the DE, $\Phi_{D E}$, the electric current, $I$, and the force, $F$, as a function of time, $t$. Cycle numbers 1 to 5 are shown on the top. (b) For the five cycles, the charge on DE at maximum stretch as a function of fourth power of the stretch. The deviation of the charge for the fifth cycle may be induced by aggregation of the carbon electrodes. The error bars are less than the size of the symbol for 2nd, 3rd and 4th cycles. Since the voltage of powder supply was constant at $3 \mathrm{kV}$, the DE capacitance varies with the fourth power of stretch as well.

The energy contributions during each cycle were calculated as follows. In one electromechanical cycle, besides the increase of the energy stored in the capacitor $\Delta E_{C}$ (Equation 2), a small part of the electric energy $\Delta E_{R}$ is also delivered to 

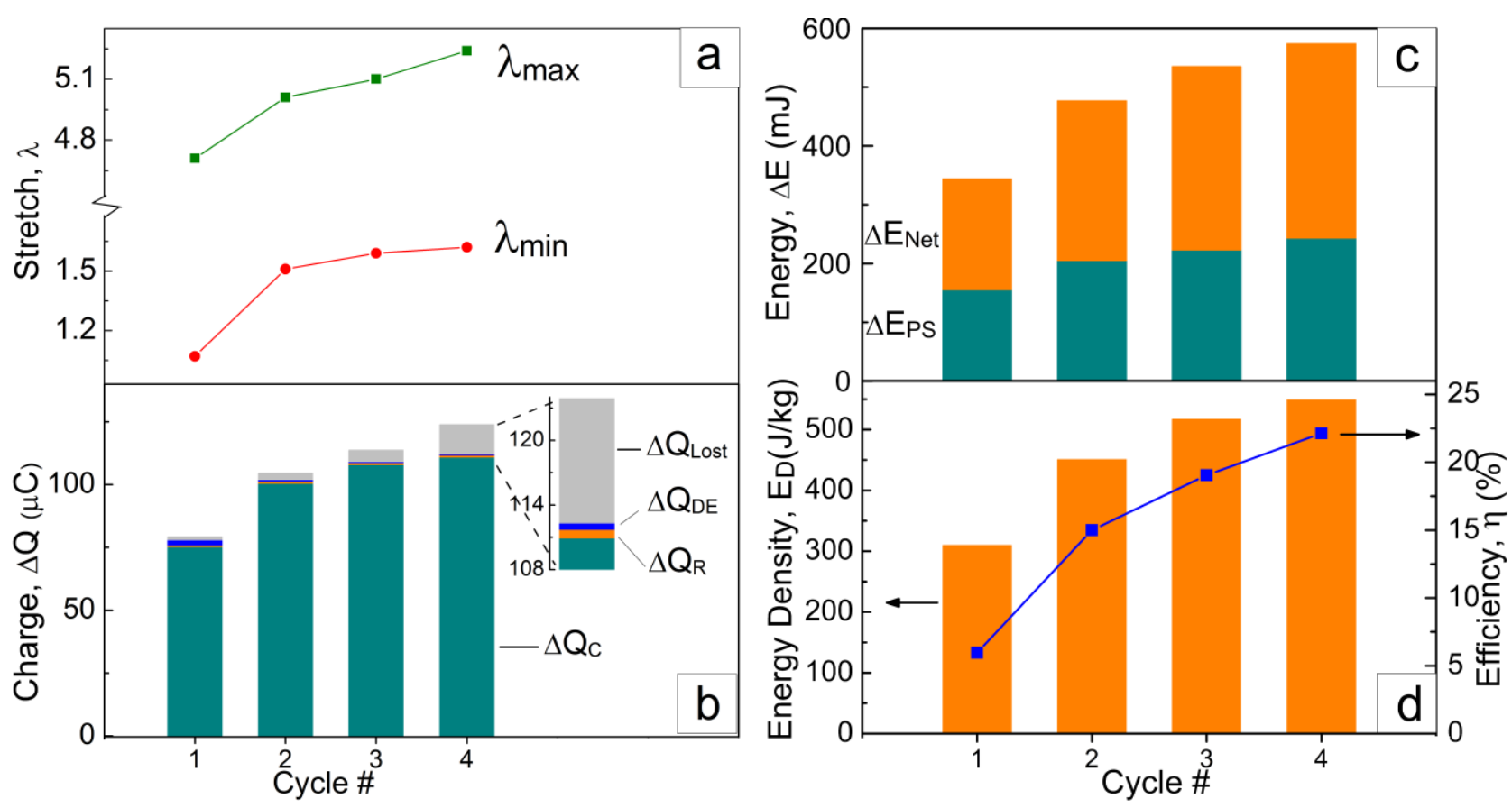

Figure 6. (a) $\lambda_{\min }$ and $\lambda_{\max }$ are the stretches of the DE at contracted state at the beginning of a cycle and at the maximum displacement in the cycle, respectively. Both are determined with video recording. (b) The electrical charges chart in which the total bar height corespond to the total charge delivered to the DE from the power supply, $\Delta Q_{P S} ; \Delta Q_{C}, \Delta Q_{R}, \Delta Q_{D E G}$, and $\Delta Q_{\text {lost }}$ are the delivered charge from DE to the capacitor, the delivered charge to the resistive load $\left(R_{1}+R_{2}\right)$, the charge increase on DE after each cycle, and the charge loss due to leakage current within the elastomer membrane, respectively. (c) The electrical energies chart, where $\Delta E_{N e t}+\Delta E_{P S}=\Delta E_{C}+\Delta E_{R}$ (eqn. 5). Since $\Delta E_{R}$ is relatively small at $3 \mathrm{~mJ}$, the total bar height correspond to the energy transferred to the harvesting capacitor. (d) The efficiency and energy density of the DE as a function of cycle number.

$R_{1}$ and $R_{2}$ (Equation 3). Taking into account the electric energy drawn from the power supply in charging the dielectric $\Delta E_{P S}$ (Equation 4), the net electrical energy harvested $\Delta E_{N e t}$ was obtained using Equation 5.

$$
\begin{aligned}
& \Delta E_{C}=C\left(\Phi_{2}^{2}-\Phi_{1}^{2}\right) / 2 \\
& \Delta E_{R}=\int_{T 0}^{T+T 0} \Phi_{D E}^{2}(t) /\left(R_{1}+R_{2}\right) d t \\
& \Delta E_{P S}=\Phi_{P S} \Delta Q_{P S}=\Phi_{P S} \int_{T 0}^{T+T 0} I d t \\
& \Delta E_{N e t}=\Delta E_{C}+\Delta E_{R}-\Delta E_{P S}
\end{aligned}
$$

Here $\Phi_{1}$ and $\Phi_{2}$ are the voltages of the collection capacitor at the start and end of a cycle, respectively, $T 0$ is the time at the start of a cycle, and $T$ is the cycle time, and $\Delta Q_{P S}$ is the charge drawn from power supply in one cycle. The energy density of conversion is defined as the net electric energy gained per cycle per unit mass of the elastomer:

$$
E_{\text {Density }}=\Delta E_{\text {Net }} / M
$$

The efficiency of conversion is defined by the ratio of the net electric energy gained to the applied mechanical work: 


$$
\eta=\Delta E_{N e t} / \mathrm{E}_{M}
$$

The results, together with the changes of number of charges and the stretches, are summarized in Fig. 6. As indicated by the graphs most of the charge drawn from the power supply was boosted up to a high voltage and then delivered to the harvesting capacitor; the charges delivered to the resistive load $\left(\mathrm{R}_{1}+\mathrm{R}_{2}\right), \Delta Q_{R}$, determined by, $\Delta Q_{R}=\int_{T 0}^{T+T 0} \Phi_{D E}(t) /\left(R_{1}+R_{2}\right) d t$, and lost due to current leakage, $\Delta Q_{\text {lost}}$, within the elastomer were relatively small in each cycle. In one cycle starting from one most constricted state to the next most constricted state, $\Delta Q_{\text {lost }}$ was calculated from the charge convervation, $\Delta Q_{\text {lost }}=\Delta Q_{P S}-\Delta Q_{C}-\Delta Q_{R}-\Delta Q_{D E}$, where $\Delta Q_{P S}$ coresponds to the total charge delivered to the DE from the power supply determined by equation (4); $\Delta Q_{C}$ is the charge delivered from DE to the capacitor determined by $C\left(\Phi_{2}-\Phi_{1}\right) ; \Delta Q_{D E}$ is the charge increase on DE after each cycle while the small charge on DE at the most constricted states were estimated through the voltage and its instantaneous capacitance with a relative dielectric constant of 4.7. ${ }^{21}$ With increasing cycle number, the energy or the charge drawn from the power source increased, which, in turn, increases the charge available for energy conversion, as evident by the increase in $\Delta E_{N e t}$. At the same time, due to relatively slow recovery time of the viscous creep of the elastomer, the mechanical energy dissipated per cycle decreased. These two factors contribute to the increase in both the efficiency and the energy density of the DEG from the 2 nd to the 4th cycle. The maximum energy density and efficiency achieved were $550 \mathrm{~J} / \mathrm{kg}$ and $22.1 \%$, respectively (Fig. 6).

The significant viscoelastic energy dissipation in the system is evident when the force-displacement curves with and without electric charge are compared (Fig. 7). In the absent of electric field, the mechanical energy dissipated, represented by the area enclosed of the dashed-red hysteresis curve, is mostly due to both the viscous deformation of the elastomer and the deformation of the mechanical loading assembly. Comparison with the curve recorded when there is an electric field (solid black curve) indicates that the areas under the two curves are comparable, demonstrating that most of the mechanical energy was lost through viscous dissipation rather than being converted to electrical energy. To quantify the amount of the energy dissipated by the mechanical loading assembly, we fixed the clips to the edge of a rigid disc of diameter $4.0 \mathrm{~cm}$ and measured the servo displacement with the same amount of maximum force as the DEG experiment. The result, which is shown by the solid blue curve in the Fig.7, indicates that the mechanical loading transfer assembly has significant energy dissipation; amounted to $23 \%$ of the total energy in the purely mechanical cycle. The loading assembly also contributes to more than $35 \%$ of the total deformation, which led to the increase in the maximum stretch as DE creeps when the number of cycle increases (Fig 6a). The rise in the maximum stretch and the buildup in the harvesting voltage increase the maximum electric field within the DE as the cycle increases, leading to either electrical breakdown or loss of tension in a relatively small number of cycles. Further developments such as using stiff metal wires and improved electronic circuitry is shown to improved energy conversion efficiencies ${ }^{22}$ but it is expected that the most significant advance is likely to come from replacing the VHB material with a more creep-resistant elastomer. 


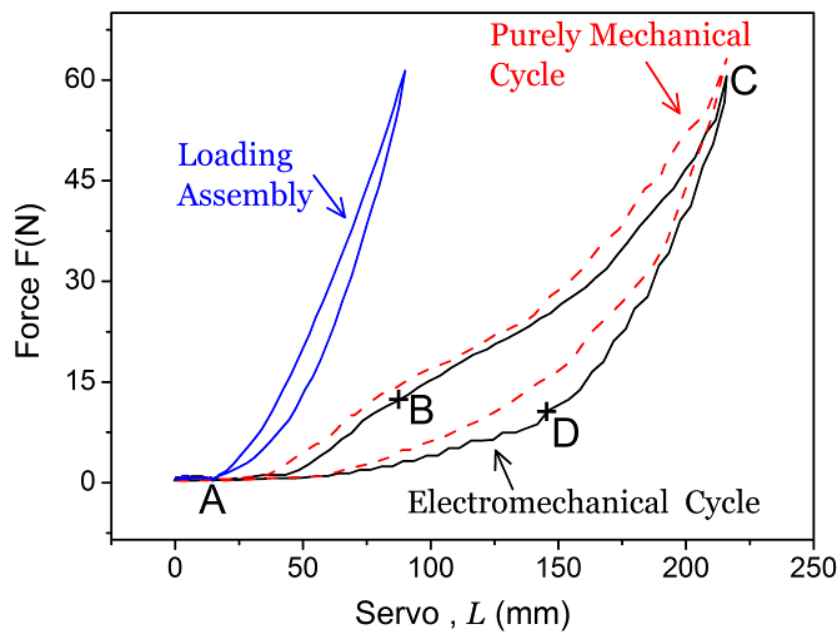

Figure 7. The force as a function of servo displacement $L$ for the third cycle of Fig. 5 (solid black curve), the third cycle of another sample without connecting to the power supply (dashed red curve), and the mechanical loading assemblies only, where the end of cotton strings were attached to the edge of a rigid disc instead of DE (solid blue curve). The area enclosed by each curve corresponds to the mechanical energy consumed during the cycle. "A, B, C, D" in the figures indicate the DE states corresponding to Fig. 4.

\section{SUMMARY}

We have demonstrated acrylic DEG under equi-biaxial mechanical loading with maximum energy density of 550 $\mathrm{J} / \mathrm{kg}$. Consistent with the simple geometrical calculation of equi-biaxial loading, the DEG capacitance scales as fourth power of the stretch, which is higher than other mechanical loading configurations. We believe that the energy conversion efficiencies can be significantly increased by replacing the DE material with a more creep-resistant elastomer.

\section{ACKNOWLEDGEMENTS}

Our research was supported by the Harvard MRSEC program of the National Science Foundation (DMR-0820484) and by Army Research Office (W911NF-09-1-0476). The authors are grateful to Tongqing Lu for discussions.

\section{REFERENCES}

[1] Pelrine, R., Kornbluh, R., Eckerle, J., Jeuck, P., Oh, S. J., Pei, Q. B., and Stanford, S., "Dielectric elastomers: Generator mode fundamentals and applications," P Soc Photo-Opt Ins, 4329, 148-156 (2001).

[2] Ashley, S., “Artificial muscles,” Sci Am, 289(4), 52-59 (2003).

[3] Chiba, S., Waki, M., Kornbluh, R., and Pelnine, R., "Innovative power generators for energy harvesting using electroactive polymer artificial muscles," Proc Spie, 6927, (2008).

[4] Jean-Mistral, C., Basrour, S., and Chaillout, J. J., "Dielectric polymer: scavenging energy from human motion," Proc Spie, 6927, (2008). 
[5] Chiba, S., and Waki, M., [Extending applications of dielectric elastomer artificial muscles to wireless communication systems] InTech, Rijeka, Croatia, 20 (2011).

[6] Chiba, S., Waki, M., Kornbluh, R., and Pelrine, R., "Current status and future prospects of power generators using dielectric elastomers," Smart Mater Struct, 20(12), (2011).

[7] Kornbluh, R. D., Pelrine, R., Prahlad, H., Wong-Foy, A., McCoy, B., Kim, S., Eckerle, J., and Low, T., "Dielectric elastomers: Stretching the capabilities of energy harvesting," Mrs Bull, 37(3), 246-253 (2012).

[8]Ihlefeld, C. M., and Qu, Z. H., "A dielectric electroactive polymer generator-actuator model: Modeling, identification, and dynamic simulation," Proc Spie, 6927, (2008).

[9] Iskandarani, Y. H., Jones, R. W., and Villumsen, E., "Modeling and experimental verification of a dielectric polymer energy scavenging cycle," Proc. of SPIE, EAPAD, 7287, 72871Y-72871Y-12 (2009).

[10] Graf, C., Maas, J., and Schapeler, D., [Energy Harvesting Cycles based on Electro Active Polymers] Spie-Int Soc Optical Engineering, Bellingham(2010).

[11] Mckay, T., O'Brien, B., Calius, E., and Anderson, I., "An integrated, self-priming dielectric elastomer generator," Appl Phys Lett, 97(6), (2010).

[12] Mckay, T., O'Brien, B., Calius, E., and Anderson, I., "Self-priming dielectric elastomer generators," Smart Mater Struct, 19(5), (2010).

[13] Kaltseis, R., Keplinger, C., Baumgartner, R., Kaltenbrunner, M., Li, T. F., Machler, P., Schwodiauer, R., Suo, Z. G., and Bauer, S., "Method for measuring energy generation and efficiency of dielectric elastomer generators," Appl Phys Lett, 99(16), (2011).

[14] Koh, S. J. A., Keplinger, C., Li, T. F., Bauer, S., and Suo, Z. G., "Dielectric Elastomer Generators: How Much Energy Can Be Converted?," Ieee-Asme T Mech, 16(1), 33-41 (2011).

[15] Kornbluh, R. D., Pelrine, R., Prahlad, H., Wong-Foy, A., McCoy, B., Kim, S., Eckerle, J., and Low, T., [From boots to buoys: Promises and challenges of dielectric elastomer energy harvesting] Spie-Int Soc Optical Engineering, Bellingham(2011).

[16] McKay, T. G., O'Brien, B. M., Calius, E. P., and Anderson, I. A., “Soft generators using dielectric elastomers,” Appl Phys Lett, 98(14), (2011).

[17] Lu, T., and Suo, Z., "Large conversion of energy in dielectric elastomers by electromechanical phase transition," Acta Mechanica Sinica, 28(4), 1106-1114 (2012).

[18] Foo, C. C., Koh, S. J. A., Keplinger, C., Kaltseis, R., Bauer, S., and Suo, Z. G., "Performance of dissipative dielectric elastomer generators,"J A Appl Phys, 111(9), (2012).

[19] Koh, S. J. A., Zhao, X. H., and Suo, Z. G., "Maximal energy that can be converted by a dielectric elastomer generator," Appl Phys Lett, 94(26), (2009).

[20] Huang, J. S., Li, T. F., Foo, C. C., Zhu, J., Clarke, D. R., and Suo, Z. G., "Giant, voltage-actuated deformation of a dielectric elastomer under dead load," Appl Phys Lett, 100(4), (2012).

[21] Kofod, G., Sommer-Larsen, P., Kornbluh, R., and Pelrine, R., "Actuation Response of Polyacrylate Dielectric Elastomers," Journal of Intelligent Materials Systems and Structures, 14(12), 787-793 (2003).

[22] Huang, J., Shian, S., Suo, Z., Clarke, D. R., "Maximizing The Energy Density of Dielectric Elastomer Generators Using Equi-Biaxial Loading," submitted to Advanced Functional Materials. 